\title{
Chattering Free Sliding Mode Observer Estimation of Liquid Water Fraction in Proton Exchange Membrane Fuel Cells
}

\author{
Julio Luna ${ }^{\mathrm{a}, *}$, Ramon Costa-Castellób ${ }^{\mathrm{b}}$,Stephan Strahl ${ }^{\mathrm{b}}$ \\ ${ }^{a}$ Chalmers University of Technology, Hörsalsvägen 11, SE-41296 Göteborg, Sweden \\ ${ }^{b}$ Institut de Robòtica i Informàtica Industrial (CSIC-UPC). C/Llorens i Artigas 4-6. 08028 Barcelona, Spain
}

\begin{abstract}
The aim of this work is to deploy and experimentally validate a model-based strategy to estimate unmeasurable variables in a proton exchange membrane (PEM) fuel cell. First, a nonlinear PEM fuel cell dynamical model is implemented and calibrated using an optimisation approach that takes real measurement data as input. Then, an advanced observation approach is developed to retrieve non-measurable data from the fuel cell. Two states are estimated in this work: the fuel cell temperature and the internal liquid water fraction. To achieve this, a model-based high-order sliding mode observer (HOSM) with chattering-free capabilities is deployed. The fuel cell temperature is measured in real time to drive the estimation error to zero in a finite amount of time. Finally, the methodology is validated using experimental data stemming from a laboratory test station, comparing the the HOSM observer with and without chattering-free gain.
\end{abstract}

Keywords: water fraction, observation, fuel cells, sliding mode, experimental validation

\section{INTRODUCTION}

As society advances towards a more technological and automated future, energy consumption is increasing exponentially. Industry, governments, and individuals are aware of the risks associated with the use of fossil fuels and the need to invest in renewable energy solutions to guarantee a sustainable future. Over the last few decades, renewable energy generation systems have witnessed an unprecedented increase in development. This growth is expected to continue over years to come, motivated by public and private investments on clean energy. It has been documented $[1,2]$ that the use of hydrogen as an energy vector can aid to satisfy the current and future energy demands without additional greenhouse gas emissions into the atmosphere.

Proton exchange membrane (PEM) fuel cells [3] are electrochemical devices that transform hydrogen into electricity in a clean manner, only generating water and heat as by-products. When compared to other fuel cell types (i.e. solid oxide fuel cells), PEM fuel cells operate at lower temperatures (usually between 40 and $80^{\circ} \mathrm{C}$ ), have shorter start-up times, better transient response, and can reach higher efficiency values. All of these advantages make PEM fuel cells an excellent candidate for a broad range of applications such as automotive, stationary combined heat and power (CHP), and portable systems. PEM fuel cells are the market leader when comparing total unit shipments to other fuel cell technologies. However, PEM fuel

\footnotetext{
*Corresponding author. Tel: +46 317721578

Email address: julio.luna@chalmers.se (Julio Luna)
}

cell technology still faces challenges. Such challenges include their relatively low durability [4], mainly caused by improper operating conditions and the high cost of the system, which is due to the associated cost of the platinum catalysts used for electrolyte membrane manufacturing. Further research needs to be done in order to address these issues.

New technological advancements to increase fuel cell durability have been achieved in the field of materials and catalysts [5]. Researchers are proposing advanced control techniques to improve the performance of fuel cells as well as improve their life expectancy [6]. For example, it has been demonstrated that the internal conditions of the fuel cell (i.e. internal water content) can affect both $[7,8]$, which highlights the importance of collecting this data when possible.

Fuel cell systems offer a wide set of measurements, such as output voltage, demanded current, and stack temperature. However, due to the construction characteristics of fuel cells, their internal parts are not accessible and sensors cannot be installed to measure variables that directly affect the operation of the system. In [9], evidence that excessive amounts of water can severely reduce the performance of a PEM fuel cell is provided. This reinforces the need to estimate the amount of water in order to operate the fuel cell efficiently. Various experimental approaches have been used to indirectly measure the water content in real time.

For example, in [9], the water content is determined from pressure drops. In [10], the water uptake from both the vapor and liquid phases is investigated experimentally, comparing the relative humidity between input and output 
gasses. In [11] an apparatus for measuring the relationship between air-water capillary pressure and water saturation in PEM fuel cells gas diffusion layers (GDL) is described. In [12] the methods of standard porosimetry and mercury intrusion porosimetry are used to generate water capillary pressure curves. In [13], water saturation is experimentally determined by weighing the GDL after imbibing or draining. Nonetheless, all of these approaches require additional sensors that can have slow and inaccurate readings, and it is possible that they cannot provide a continuous measure, invalidating their use for control deployment. Moreover, additional sensors increases the cost of the system. Using model-based observers allows for the estimation of the water content using sensors that are already available in the system.

Aside from the technical difficulties to measure the internal conditions of a fuel cell, another challenge these systems face is the high amount of uncertainties when being operated. Uncertainty can originate from a varied array of sources, such as the external power demand (i.e. automotive applications with different driving profiles) or from the fuel cell itself in the form of membrane ageing during time, accumulated liquid water, and starvation scenarios, among others. These uncertainties have to be taken into account when selecting control and estimation strategies. In this regard, sliding mode control (SMC) has been proved accurate and robust with respect to internal and external disturbances $[14,15,16]$. However, the main drawback of classic SMC is its propensity to chattering along the sliding surface when the measured variables have associated noise, which is common in electric power generation applications. To solve this, high-order implementations of classic SMC schemes have been proved to reduce chattering $[17,18]$.

The motivation of this paper is to establish an experimentally validated model-based estimation strategy that can aid to obtain unmeasurable internal variables that affect the performance of PEM fuel cells. Furthermore, uncertainties in the form of measurement noise will be considered when analysing the proposed observer. To achieve these goals, a nonlinear high-order sliding mode (HOSM) observer will be developed to estimate the fuel cell temperature and the internal liquid water fraction of an open cathode PEM fuel cell. In addition, a chattering-free HOSM (CHOSM) observer will be proposed to further improve the chattering rejection capabilities of the standard HOSM observer approach. Both observation strategies are based on a PEM fuel cell model that has been experimentally validated in the literature [19]. This model is calibrated to account for the possible ageing of the system using experimental data. Finally, both observation approaches are validated using experimental data stemming from a laboratory test station.

Thus, the main contributions of this paper can be summarised as follows:

1. A model-based methodology to estimate the liquid water fraction from measurable variables on an open cathode PEM fuel cell.

2. Deployment of two observers with and without adaptive gain strategy enhancement to prove the reduction of chattering due to noisy measurement data.

3. Experimental validation of the conceptual contributions presented in this paper.

The rest of the paper is organised as follows. The experimental PEM fuel cell test station and the open-cathode fuel cell simulation model are introduced in Section 2. Then, the model parameters are calibrated using experimental data in Section 3. The observation problem and HOSM and CHOSM observers are developed in Section 4. Experimental results for a given laboratory test study are presented and discussed in Section 5, comparing the HOSM and CHOSM observers with a conventional SMC approach to show the improvements of the proposed strategy. Finally, Section 6 summarises the results of this paper and proposes research lines for future work.

\section{EXPERIMENTAL SETUP}

Fuel cell systems are supported by the balance of plant (BOP) devices, such as compressors, humidifiers, and line heaters. All of these elements allow the control of the most relevant variables. However, they also make fuel cell systems complex and expensive to operate. To minimise these problems, open cathode fuel cells have been suggested. These fuel cells do not use most of the aforementioned ancillary elements and therefore, are much cheaper and simpler to operate. This fact makes open cathode fuel cells ideal to test novel control and observation strategies. One of these fuel cells is the Horizon Fuel Cells Technologies $\mathrm{H}-100$, which has a nominal power of $100 \mathrm{~W}$. This fuel cell will be used in this work to perform the experiments and validate the proposed observers.

\subsection{System description}

Figure 1 shows the experimental station used to run the experiments. Figure 2 contains a scheme of the H-100 stack, which consists of 20 cells $\left(n_{\text {cell }}=20\right)$. A speed regulated fan is in charge of the temperature control and also feeds the fuel cell cathode with oxygen, taken directly from the air. The anode of the H-100 is fed with dry hydrogen that comes from a pressurised tank. The supply pipe is completed with a valve that allows to maintain the pressure and to perform purges. Finally, an air speed sensor, E75 E+E Elektronik, is included in the system.

The small number of complementary elements makes open cathode fuel cells sensitive to environmental conditions and their performance can decrease in the presence of internally accumulated liquid water. Thus, it is compelling to be able to estimate the amount of liquid water fraction in order to improve the fuel utilisation and global efficiency of the system. 


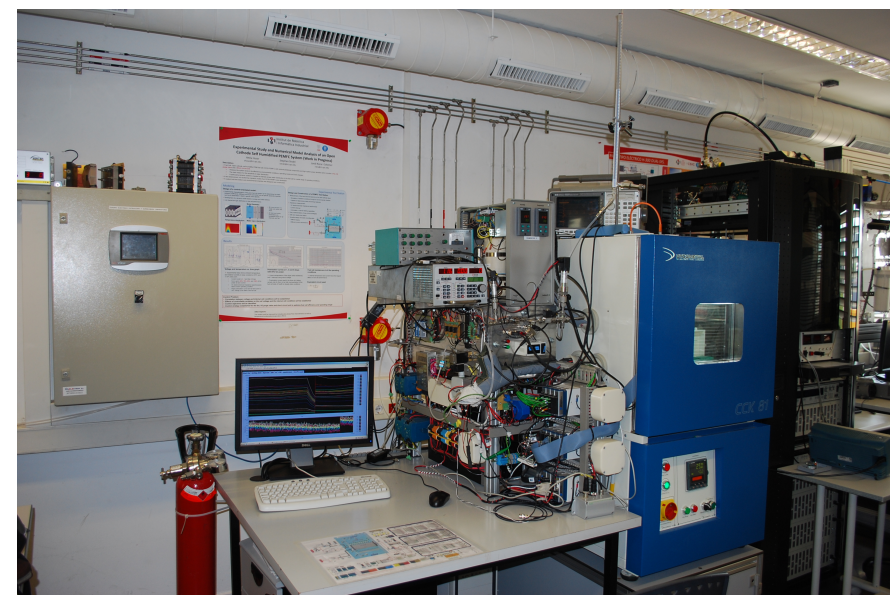

Figure 1: Experimental station used to validate the observers

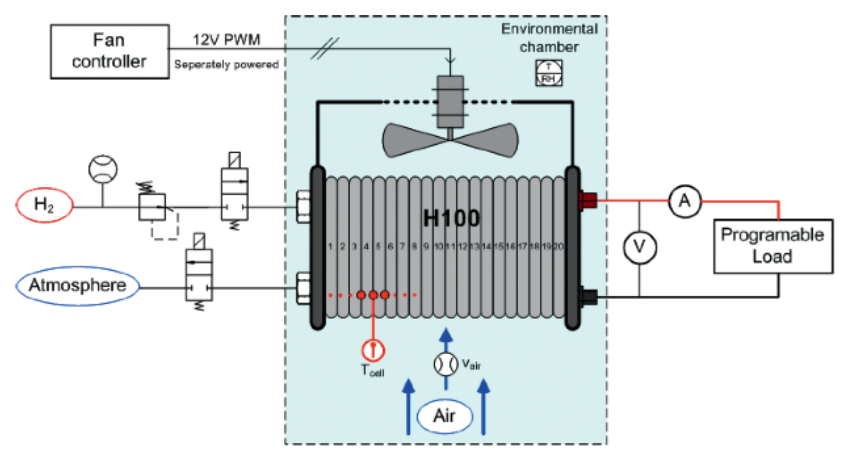

Figure 2: H-100 experimental set-up diagram

From the current measurement, an algorithmic approach to estimate the liquid water fraction is developed in Section 4 and deployed in Section 5. To accomplish this, a suitable mathematical model of the experimental station is needed for the analysis of the results and to validate the observers. In the following section, the model for the H-100 that will be used to develop and implement the observers is presented.

\subsection{Open cathode fuel cell model}

The literature contains open cathode fuel cell models with multiple levels of complexity and detail. In this paper, an experimentally validated $\mathrm{H}-100$ model [19] is going to be used. This model has been able to accurately reproduce the behaviour of an open cathode PEM fuel cell with a reduced number of states. Based on this, it will be possible to design an observation strategy that is both accurate and feasible to implement in real time.

The H-100 model in [19] is described by the next set of equations:

$$
\begin{aligned}
\dot{T}_{f c}= & K_{1} \cdot I_{f c}-K_{1}^{\prime} \cdot I_{f c} \cdot V_{f c} \\
& +\left(K_{2} \cdot T_{a m b}-K_{2} \cdot T_{f c}\right) u_{a i r} \\
\dot{s}_{f c}= & F_{g e n}\left(I_{f c}\right)-F_{\text {evap }}\left(T_{f c}, s_{f c}\right)-F_{d i f f}\left(s_{f c}\right) \\
V_{f c}= & K_{7}-K_{6} \cdot I_{f c} \\
& -K_{5} \cdot T_{f c} \cdot \log \left(f_{a}\left(T_{f c}, s_{f c}, I_{f c}\right)\right)
\end{aligned}
$$

The model state variables are the fuel cell temperature $\left(T_{f c}\right)$ and liquid water fraction $\left(s_{f_{c}}\right)$. This last variable corresponds to the proportion of total volume of empty space in the porous structure of the cathode catalyst layer (CCL) that is occupied by liquid water [20]. The model is described by a set of constant parameters, denoted by $K_{i}$ $(i \in[1, \ldots, 7])$, that can be obtained from physical constants and the construction standards of the fuel cell. Appendix A shows the equations required to calculate all of these parameters. Additionally, the model has an output variable, $V_{f c}$, which corresponds to the fuel cell voltage.

Equations (1)-(3) contain variables that are treated as exogenous variables. These are the current $\left(I_{f c}\right)$, the ambient temperature $\left(T_{a m b}\right)$, and the air velocity $\left(u_{a i r}\right)$. The current is fixed by the load fed by the fuel cell. Note that the provided electrical power is obtained as $P_{f c}=V_{f c} \cdot I_{f c}$. The ambient temperature $T_{a m b}$ is usually treated as a measurable disturbance, while $u_{\text {air }}$ takes the control action role (it can be manipulated by changing the fan turning speed).

The liquid water fraction in Equation (2) is defined as the equilibrium between generated $\left(F_{\text {gen }}\right)$, evaporated $\left(F_{\text {evap }}\right)$, and diffused $\left(F_{\text {diff }}\right)$ water in the PEM fuel cell cathode. These terms can be computed as follows [19]:

$$
\begin{aligned}
F_{g e n}\left(I_{f c}\right) & =K_{3} \cdot I_{f c}, \\
F_{\text {evap }}\left(T_{f c}, s_{f c}\right) & =f_{p}\left(T_{f c}\right) \cdot \frac{s_{f c}}{K_{s}}, \\
F_{\text {diff }}\left(s_{f c}\right) & =\frac{J_{\text {diff }}}{K_{s}},
\end{aligned}
$$

being $J_{\text {diff }}$ the diffusive flux and $K_{s}$ the water retention constant in the CCL respectively. The full equations for these terms are

$$
\begin{aligned}
J_{d i f f}= & \frac{K_{d i f f}}{d_{e f f}} \cdot s_{f c}^{3} \cdot\left(3.79 s_{f c}^{2}-4.24 s_{f c}+1.42\right) \\
& \cdot\left(s_{f c}-s^{o p t}\right) \\
K_{s}= & \epsilon_{e f f} \cdot \rho_{l} \cdot d_{C C L} \cdot K_{\text {sorp }},
\end{aligned}
$$

where $K_{\text {diff }}$ is the diffusion time constant and $d_{\text {eff }}$ the effective thickness of the diffusion media. The CCL thickness and effective porosity are described by $d_{C C L}$ and $\epsilon_{e f f}$ respectively. Finally, $\rho_{l}$ is the liquid density of water and $K_{\text {sorp }}$ the sorption time constant.

Although a direct inspection of the proposed model can lead to the conclusion that it is a simple 2-state model, in fact, it is highly nonlinear [19]. This makes its analysis quite difficult, as demonstrated by [21]. Appendix A contains the highly nonlinear algebraic expressions $f_{a}$ and 
Table 1: Model parameters used in the calibration

\begin{tabular}{lrrr} 
Parameter & Initial value & Optimised value & Units \\
\hline$R_{\text {ohm }}$ & 0.300 & 0.140 & $\Omega$ \\
$K_{\text {sorp }}$ & 360.000 & 26.383 & - \\
$K_{\text {evap }}$ & $1.400 \cdot 10^{-2}$ & $1.733 \cdot 10^{-2}$ & - \\
$K_{\text {diff }}$ & $2.127 \cdot 10^{-4}$ & $1.081 \cdot 10^{-4}$ & - \\
\hline
\end{tabular}

$f_{p}$

To reduce complexity, the proposed model can be partially linearised and simplified by using the following definition:

$$
u_{a i r} \triangleq \frac{\nu}{K_{2}\left(T_{a m b}-T_{f c}\right)} .
$$

Note that $\nu$ can be thought of as the thermal power extracted from the fuel cell.

Taking (9) into account, the fuel cell model can be rewritten as follows:

$$
\begin{aligned}
\dot{T}_{f c}= & K_{1} \cdot I_{f c}-K_{1}^{\prime} \cdot I_{f c} \cdot V_{f c}+\nu \\
\dot{s}_{f c}= & F_{g e n}-F_{\text {evap }}-F_{d i f f} \\
V_{f c}= & K_{7}-K_{6} \cdot I_{f c} \\
& -K_{5} \cdot T_{f c} \cdot \log \left(f_{a}\left(T_{f c}, s_{f c}, I_{f c}\right)\right) .
\end{aligned}
$$

\section{EXPERIMENTAL MODEL CALIBRATION}

Before designing the observers, the fuel cell model described in Section 2.2 is adjusted using experimental data. A multi-variable optimisation is used to match the output voltage in (12) with the experimental value. The variables to be tuned are selected from fuel cell parameters related to the water dynamics described in Equation (11). The variables chosen are the ohmic stack resistance $R_{o h m}$, the sorption time constant $K_{\text {sorp }}$, the evaporation time constant $K_{\text {evap }}$, and the diffusion time constant $K_{\text {diff }}$. The selection of this set of variables is due to their relation with the state-of-health of the fuel cell [19], varying with the ageing of the system and resulting in a decreased output voltage over time. Table 1 summarises the optimisation variables, including the initial values, obtained from the validated model presented in [19].

The output voltage given by the simulation model from Equation (12) is assumed to be

$$
V_{f c, \text { simulated }} \triangleq f\left(\mathbf{p}_{k}\right)
$$

where $\mathbf{p}_{k}$ is the vector containing the optimisation parameters from Table 1 . In order to solve the optimisation problem and obtain the set of $\mathbf{p}_{k}$ parameters, a least squares minimisation method is carried out. This strategy takes the measured fuel cell voltage $\left(V_{f c, \text { measured }}\right)$ from the experimental test station and compares it to the fuel cell output voltage in Equation (13). This can be expressed as a minimisation problem of the form

$$
\min _{\mathbf{p}_{k}} \sqrt{\sum_{k=1}^{N}\left(V_{f c, \text { measured }}^{(k)}-V_{f c, \text { simulated }}^{(k)}\right)^{2}} .
$$

An iterative procedure over $N$ samples is used to find the best-fitting $\mathbf{p}_{k}$ value set. Once a minimum is found, the iterative procedure stops and updates the model parameters with the set of optimal values. Figure 3 shows the experimental data (in blue) used to calibrate the model. This experimental data consists of two voltage steps distributed over 3000 seconds in order to properly characterise the slow temperature dynamic of the model.

As shown in Figure 3(a), before the calibration there is a noticeable difference between the experimental data and the simulated voltage. This is due to the ageing of the test station, producing an outdated set of model parameters that need to be updated. Once the optimisation problem is solved and the parameters are recalculated, it can be seen in Figure 3(b) that the fitting is significantly improved. The updated set of parameter values is included in the third column of Table 1 .

a)

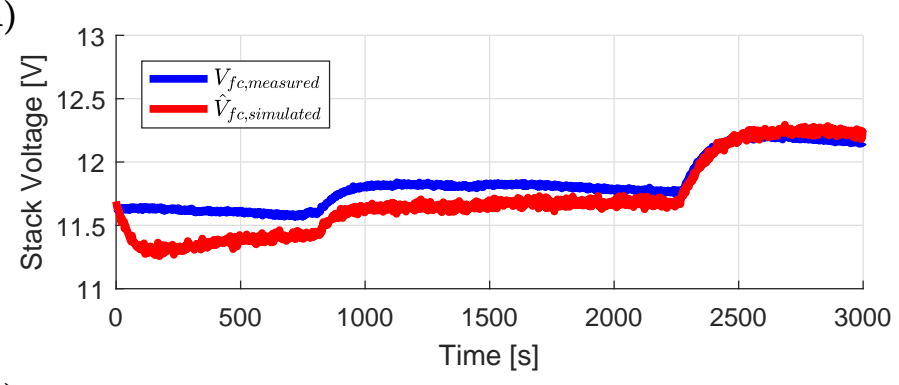

b)

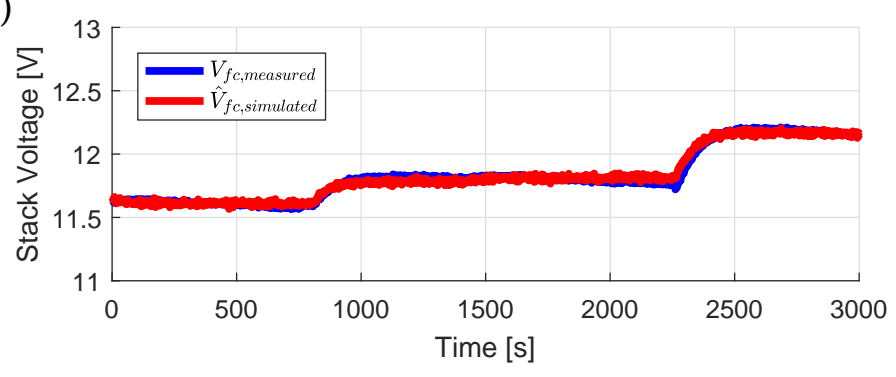

Figure 3: Simulated and measured fuel cell voltage output before (a) and after (b) the calibration procedure

Even after calibration, some discrepancies between the simulation model voltage output and the measured experimental data can be detected. These differences come from measurement noise and dynamics that are not included in the fuel cell model. An example of dynamics not captured by the model is the voltage drop that occurs in the experimental data on the second voltage step around the 2250 second mark. Nevertheless, these fast dynamics do not affect the long-term performance and accuracy of the simulation model, making the calibrated model suitable for observers design. Therefore, the open cathode PEM fuel 
cell model will be used to design the observation strategy in the following sections, providing additional robustness against model uncertainties and measurement noise thanks to the HOSM capabilities included in the proposed approach.

\section{HOSM OBSERVER}

Applying the observer structure described in [22] to the fuel cell model presented in Section 2.2, the following observer is obtained:

$$
\begin{aligned}
& \dot{\hat{x}}_{1}=K_{1} \cdot I_{f c}-K_{1}^{\prime} \cdot I_{f c} \cdot V_{f c}+\nu+g_{1}(\hat{\mathbf{x}}) \cdot u_{1}, \\
& \dot{\hat{x}}_{2}=K_{3} \cdot I_{f c}-f_{p}\left(\hat{x}_{1}\right) \cdot \frac{\hat{x}_{2}}{K_{s}}-\frac{J_{d i f f}}{K_{s}}+g_{2}(\hat{\mathbf{x}}) \cdot u_{2}, \\
& \hat{y}=h(\hat{\mathbf{x}})=\hat{x}_{1},
\end{aligned}
$$

being $\hat{\mathbf{x}} \triangleq\left[\hat{x}_{1}, \hat{x}_{2}\right], \hat{x}_{1} \triangleq \hat{T}_{f c}$, and $\hat{x}_{2} \triangleq \hat{s}_{f c}$. The fuel cell temperature is used as the measured variable as denoted by $(15 \mathrm{c})$. Moreover, the output fuel cell voltage $V_{f c}$ is also measured. However, $V_{f c}$ is not included in Equation (15c) since it is not a state variable. Functions $g_{1}, g_{2}$, and the corrective terms $u_{1}$ and $u_{2}$ need to be formulated in order to make the observer converge in finite time to the expected state values [22].. To achieve this, an observability analysis of the fuel cell model is conducted in the following section.

\subsection{Observability analysis}

In linear systems, observability is a global property, whereas in nonlinear systems it is a local property. This can be verified in terms of the observability matrix $\mathcal{O}$ rank, which is defined as follows [22]:

$$
\mathcal{O}(\mathbf{x})=\frac{\partial}{\partial \mathbf{x}}\left[\begin{array}{c}
h(\mathbf{x}) \\
L_{f(\mathbf{x})} h(\mathbf{x}) \\
\vdots \\
L_{f(\mathbf{x})}^{n-1} h(\mathbf{x})
\end{array}\right],
$$

where $n$ is the model order and $L_{f(\mathbf{x})} h(\mathbf{x})$ stands for the Lie derivative of the output vector $h$ along the vector field $f(\mathbf{x}) \in \mathbb{R}^{n}$. It can be computed as [23]:

$$
L_{f(\mathbf{x})} h(\mathbf{x})=\frac{\partial h(\mathbf{x})}{\partial \mathbf{x}} f(\mathbf{x}) .
$$

And the $k$-th Lie derivative:

$$
L_{f(\mathbf{x})}^{k} h(\mathbf{x})=\frac{\partial\left(L_{f(\mathbf{x})}^{k-1} h(\mathbf{x})\right)}{\partial \mathbf{x}} f(\mathbf{x}) .
$$

Using Equations (17) and (18) in Equation (15), the observability matrix is obtained:

$$
\mathcal{O}(\hat{\mathbf{x}})=\left[\begin{array}{cc}
1 & 0 \\
\xi(\hat{\mathbf{x}}) & \gamma(\hat{\mathbf{x}})
\end{array}\right]
$$

being $\xi$ and $\gamma$ state functions obtained using Lie algebra. The rank of $\mathcal{O}$ depends only on $\gamma(\hat{\mathbf{x}})$ and the value of $\hat{\mathbf{x}}$ [22] at a given time. Note that the determinant of $\mathcal{O}$ is equal to $\gamma(\hat{\mathbf{x}})$. According to (19) :

$$
\gamma(\hat{\mathbf{x}})=-0.01 \frac{I_{f c} \cdot K_{1}^{\prime} \cdot K_{5} \cdot \hat{x}_{1} \cdot \exp ^{\frac{0.01 \cdot \hat{x}_{2}}{s^{o p t}}}}{\left(\exp \frac{0.01 \cdot \hat{x}_{2}}{s^{o p t}}-1\right) \cdot s^{o p t}},
$$

being $s^{\text {opt }}$ the optimal water content for the H-100 PEM fuel cell (see Table A.2) [21].

For the system under study, the observability condition becomes:

$$
\gamma(\hat{\mathbf{x}}) \neq 0
$$

As $K_{1}^{\prime}, K_{5}$, and $s^{\text {opt }}$ are non-zero parameters (see Appendix A), the full-range condition for $\mathcal{O}$ is fulfilled when $x_{1}, x_{2}$, and $I_{f c}$ are not equal to zero.

Once the observability is guaranteed, functions $g_{1}$ and $g_{2}$ in Equation (15) are obtained as follows [22]:

$$
\mathbf{g}(\hat{\mathbf{x}})=\left[\begin{array}{l}
g_{1}(\hat{\mathbf{x}}) \\
g_{2}(\hat{\mathbf{x}})
\end{array}\right]=\mathcal{O}(\hat{\mathbf{x}})^{-1}\left[\begin{array}{ll}
0 & 1
\end{array}\right]^{T},
$$

which results in

$$
\begin{aligned}
g_{1}(\hat{\mathbf{x}}) & =0, \\
g_{2}(\hat{\mathbf{x}}) & =-100 \frac{s^{o p t} \cdot\left(\exp \frac{0.01 \cdot \hat{x}_{2}}{s^{o p t}}-1\right)}{I_{f c} \cdot K_{1}^{\prime} K_{5} \hat{x}_{1} \cdot \exp ^{\frac{0.01 \cdot \hat{x}_{2}}{s^{o p t}}} .}
\end{aligned}
$$

Finally, considering $g_{1}$ and $g_{2}$, the observer described in Equation (15) is expressed as

$$
\begin{aligned}
\dot{\hat{x}}_{1}= & K_{1} \cdot I_{f c}-K_{1}^{\prime} \cdot I_{f c} \cdot V_{f c}+\nu, \\
\dot{\hat{x}}_{2}= & K_{3} \cdot I_{f c}-f_{p}\left(\hat{x}_{1}\right) \cdot \frac{\hat{x}_{2}}{K_{s}}-\frac{J_{d i f f}}{K_{s}} \\
& \quad-100 \frac{s^{o p t} \cdot\left(\exp \frac{0.01 \cdot \hat{x}_{2}}{s^{o p t}}-1\right)}{I_{f c} \cdot K_{1}^{\prime} K_{5} \hat{x}_{1} \cdot \exp \frac{0.01 \cdot \hat{x}_{2}}{s^{o p t}}} \cdot u_{2}, \\
\hat{y}= & \hat{x}_{1} .
\end{aligned}
$$

\subsection{Corrective action design}

The PEM fuel cell model in Equations (10)-(12) considers two measured variables: $T_{f c}$ and $V_{f c}$ and two derived parameters, $\nu^{1}$ and $I_{f c}$. In this paper, the observation error $e_{y}$ is expressed as the difference between the measured fuel cell temperature $T_{f c}$ and the estimated variable $\hat{T}_{f c}$ :

$$
e_{y}=T_{f c}-\hat{T}_{f c}
$$

From Equation (26) the sliding surface is selected as:

$$
\sigma=e_{y}
$$

\footnotetext{
${ }^{1} \nu$ can be computed from $u_{a i r}, T_{f c}$ and $T_{a m b}$
} 
and the sliding manifold $\ell$ is

$$
\ell=\dot{\sigma}-k \sigma,
$$

where $k$ is a positive parameter used to improve the convergence to the sliding surface.

The purpose of the observer is to reduce the difference in Equation (26) to null in the shortest time possible (ideally a finite amount). To achieve this, a second-order quasicontinuous term $u_{2}$ is chosen as the corrective signal [24]:

$$
u_{2}=-\beta \cdot\left(\frac{\dot{\sigma}+|\sigma|^{1 / 2} \cdot \operatorname{sign}(\sigma)}{|\dot{\sigma}|+|\sigma|^{1 / 2}}\right),
$$

being $\beta$ the gain magnitude of the observer.

Any possible uncertainties in Equation (25) are assumed to be compensated with the corrective input in Equation (29). A side effect of this implementation is chattering appearing on $u_{2}$ [25], which can introduce additional chattering to the estimation variable. In the next section, a chattering-free solution for the observer gain $\beta$ is developed. In Section 5, the results with and without chattering will be compared and discussed.

\subsection{HOSM observer with chattering-free gain}

As mentioned, the design of the observer in Equation (25) can lead to chattering when using a fixed-gain magnitude in the corrective input (29). To deal with this issue, an adaptive gain is proposed in this section: as the sliding manifold in Equation (28) approaches to zero, the observer gain will also be reduced to minimise the effect of chattering in the correction input $u_{2}$.

The strategy of using an adaptive gain for SMC implementations was introduced in [26], where the gain is defined as a dynamic state of the form:

$$
\dot{\hat{\beta}}= \begin{cases}\theta \cdot(-\rho \hat{\beta}+\|\sigma-\wp\|), & \text { if } \ell \neq 0, \\ 0, & \text { otherwise. }\end{cases}
$$

Note that Equation (30) includes the sliding manifold in (28) as the condition to choose between the two possible gain outputs. If the sliding manifold is different than zero, the derivative of the adaptive gain is a function of the positive constants $\theta$ and $\rho$, which have to be properly tuned to guarantee a meaningful settling time of the observer. Moreover, $\wp$ is a small positive value, assuring that $\hat{\beta}$ can remain at a minimal value while maintaining certain state estimation effectiveness even in the presence of model or measurement uncertainties [26].

\section{EXPERIMENTAL RESULTS}

The observer with chattering-free capabilities uses the adaptive $\hat{\beta}$ gain described in Equation (30) with the parameters $\theta=5 \cdot 10^{-2}, \rho=1$, and $\wp=1 \cdot 10^{-3}$. It will be compared to the standard HOSM observer and a classic SMC estimator implementation [27], both with a fixed gain $\beta=5 \cdot 10^{-2}$. With the selected CHOSM parameters, the time constant in Equation (30) is equal to 20 seconds, which will result in a settling time of approximately 80 seconds, consistent with the measured temperature time constant. The observers are initialised with the following estimated state values: $\hat{\mathbf{x}}(0)=\left[\hat{x}_{1}(0), \hat{x}_{2}(0)\right]=[298 K, 0]$.

\subsection{Experimental case study}

To test the proposed observation strategy, an experiment scenario is planned on the test station. This experiment consists of a constant demanded current of $4 \mathrm{~A}$ and a given PWM duty cycle profile for the cooling fan, shown in the right-axis of Figure 5. The relation between the PWM duty cycle and the system input $u_{\text {air }}$ in Equation (9) is described by a fourth degree polynomial [21]:

$$
\begin{aligned}
u_{\text {air }}= & -9.1685 \cdot \mathrm{PWM}_{\text {fan }}^{4}+17.711 \cdot \mathrm{PWM}_{\text {fan }}^{3} \\
& -9.1117 \cdot \mathrm{PWM}_{\text {fan }}^{2}+1.7135 \cdot \mathrm{PWM}_{\text {fan }} \\
& -0.1013 .
\end{aligned}
$$

The change in the fan speed will affect the fuel cell temperature and therefore the liquid water fraction and voltage output of the system. The experiment is performed inside an environmental chamber (see Figure 2) at a constant ambient temperature of $298 \mathrm{~K}$.

\subsection{Results and discussion}

As introduced in Section 4, the measured temperature of the PEM fuel cell is used to design the sliding surface in Equation (27). Figure 4(a) shows the evolution of the fuel cell temperature $\left(T_{f c}\right)$ and the SMC, HOSM, and CHOSM observers estimations $\left(\hat{T}_{f c, S M C}, \hat{T}_{f c}\right.$, and $\left.\hat{T}_{f c, c f}\right)$. In a similar way, Figure 4(b) presents the dynamic response of the modelled liquid water fraction $\left(s_{f c}\right)$ and the estimation obtained from the observers $\left(\hat{s}_{f c, S M C}, \hat{s}_{f c}\right.$, and $\left.\hat{s}_{f c, c f}\right)$.

As shown in Figure 4(a), the three observers are capable of estimating the temperature with minimum error throughout the experiment, even in the presence of changes in the cooling fan speed. This was to be expected, since $T_{f c}$ is the measured variable used to obtain the observation error $e_{y}$ in Equation (26). Convergence is achieved after 50 seconds, mainly due to the slow temperature dynamics. In Figure 4(b), the chattering problems that arise from the SMC approach are clearly noticeable. While both the HOSM and CHOSM observers are able to reduce the chattering after convergence to $s_{f c}$, the observation error introduced by the SMC makes it unsuitable for future control strategies, justifying the use of higher order approaches. Subsequently, from this point forward the analysis of the experimental results will only consider the HOSM and CHOSM implementations.

Using the estimated temperature and liquid water saturation, an estimation of the fuel cell voltage $\hat{V}_{f c}$ can be derived using Equation (12). The left-axis of Figure 5 shows the measured output voltage and the estimated fuel cell voltage for the HOSM and the CHOSM observers $\left(\hat{V}_{f c}\right.$ 
a)

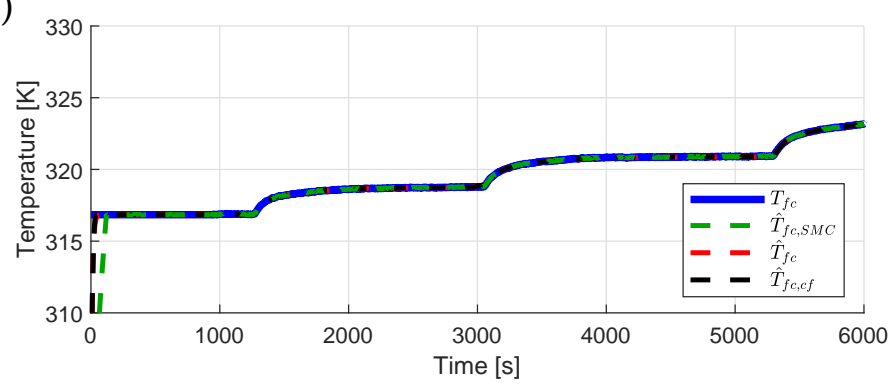

b)

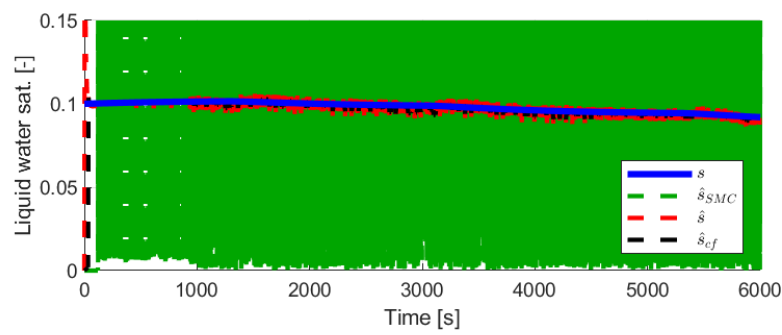

Figure 4: Temperature (a) and liquid water fraction (b) evolution and observer estimations

and $\left.\hat{V}_{f c, c f}\right)$. The output voltage shows the dynamic response to the cooling fan speed changes. Moreover, after $t=1000 \mathrm{~s}$, the fuel cell anode is purged at a constant frequency. These purges generate voltage drops of unknown values that affect the observed variables, as seen previously in Figure 4.

Figure 5 shows that both observers achieve convergence to the measured voltage value. As previously discussed, the convergence time is governed by the time constants on the corrective inputs. Once the sliding manifold reaches $\ell \approx 0$, both the HOSM and CHOSM observers maintain convergence, until the anode purges begin to happen. When this occurs, the HOSM observer is not capable of recovering the value of the voltage due to the chattering effect in the estimated states. Meanwhile, the CHOSM observer chattering effect is reduced due to the better estimation of $T_{f c}$ and $s_{f c}$.

Figure 6 shows the evolution of the state estimation error for the fuel cell temperature and the water content. Both states quickly converge to the vicinity of zero and remain there during the rest of the experiment, even when the fan speed changes. Slight deviations can be observed in both observation approaches, mainly due to the nonmodelled purges present in the output voltage measurement and other modelling errors of the observer. To solve this, in future stages of the research, an additional voltage state can be included in the observer to take into consideration new dynamics that affect the fuel cell output.

The behaviour of the HOSM $\left(u_{2}\right)$ and CHOSM $\left(u_{2, c f}\right)$ corrective inputs during the experiment are represented in Figure 7(a). These corrective actions drive the temperature measurement estimation error in Equation (26) to zero in the shortest amount of time possible. As it can be noticed, $u_{2}$ maintains a sliding dynamic bounded by the

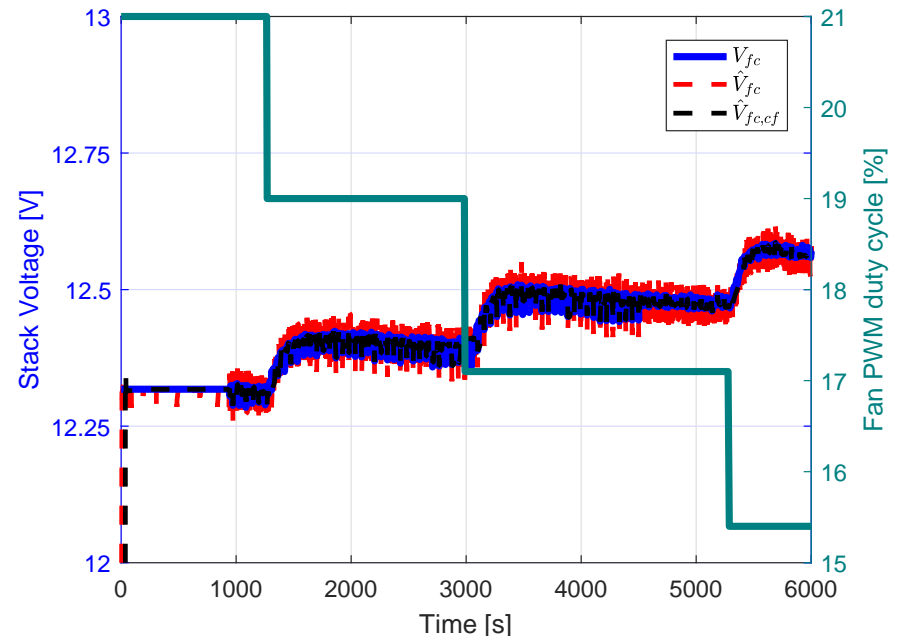

Figure 5: Voltage output and estimation (left) and cooling fan PWM duty cycle (right) during the experiment

a)

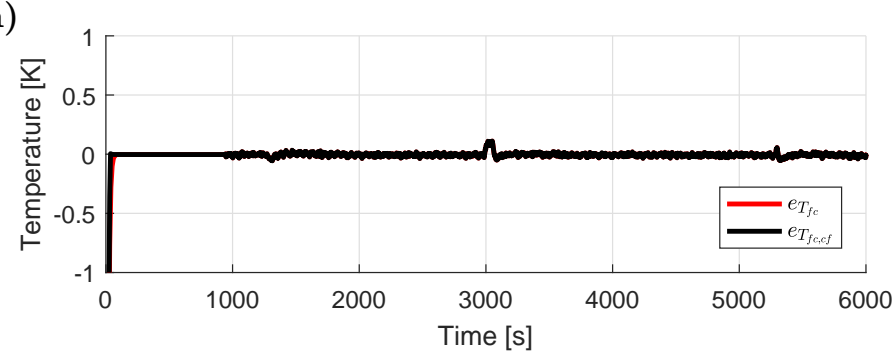

b)

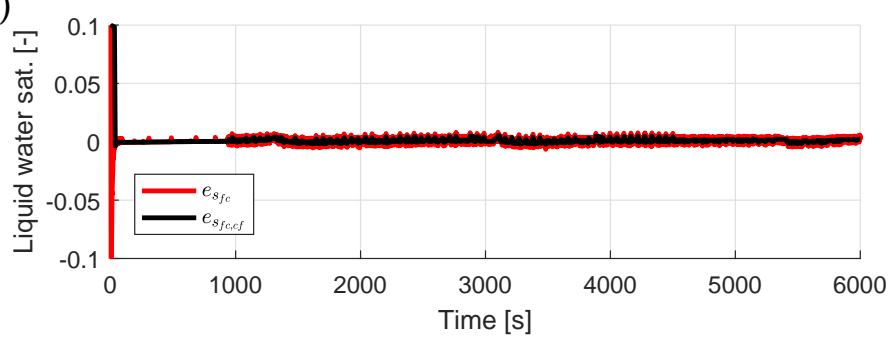

Figure 6: State estimation errors for the temperature (a) and the liquid water fraction (b)

maximum value of $\beta$. In the case of the CHOSM observer, the corrective action $u_{2, c f}$ is limited by the adaptive gain law from Equation (30). This guarantees the tracking of the sliding surface while maintaining the chattering to a minimum. The value of the adaptive gain $\hat{\beta}$ is shown in Figure 7(b) and compared to the constant value $\beta$ for the HOSM observer. As it reaches the sliding surface $\sigma$, the gain $\hat{\beta}$ decreases to reduce the chattering effect, and reacts to the presence of the non-modelled purges by increasing the gain to reduce the effect of these perturbations in the estimation process.

\subsection{Robustness analysis}

An important aspect to study in an observer is its capability to return precise estimations even in the presence of uncertainties. While it can be argued that the inclusion of the non-modelled purges in the experimental analysis is 
a)

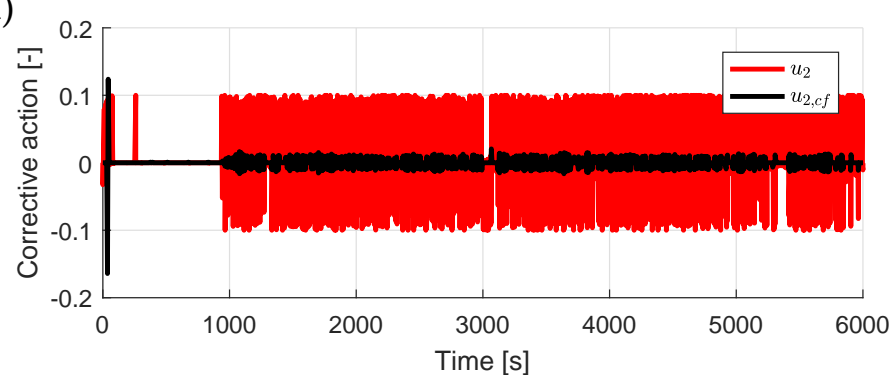

b)

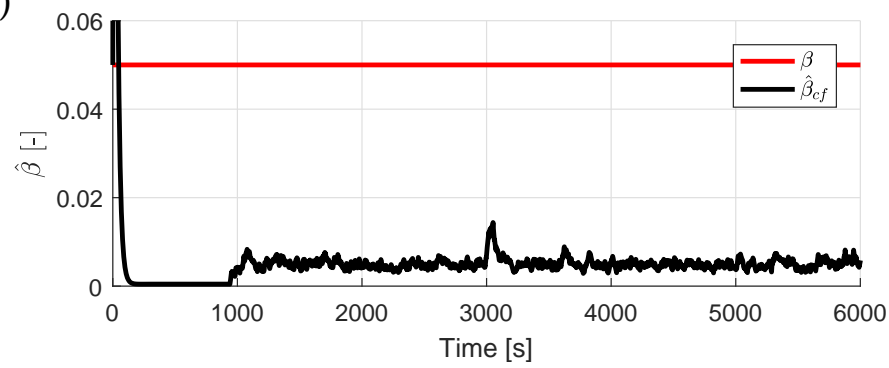

Figure 7: Corrective action (a) and observer gain evolution (b) during the experiment

sufficient proof of robustness, in this section an additional robustness test of the HOSM and CHOSM observers is evaluated. In particular, to test the proper convergence with the experimental data, the measurement noise of the fuel cell measured temperature is increased with a bandlimited white noise with noise power equal to 0.01 . Figure 8 shows that the observers are still able to track the states satisfactorily with only a slight increase in the liquid water fraction estimation noise. This is an expected outcome as the estimated value uses the measured temperature as the sliding surface for the correction action. While the CHOSM observer estimation noise is lower, due to the chattering-free capabilities of its adaptive gain, it is worth mentioning that the convergence time is delayed when compared to the HOSM observer.

\section{CONCLUSIONS AND FUTURE WORK}

In this paper, a nonlinear chattering-free HOSM observer to estimate the temperature and internal liquid water fraction in the cathode catalyst layer of a PEM fuel cell has been developed. The original HOSM strategy was enhanced using an adaptive gain approach. Both solutions were deployed and validated using experimental data from a test station, comparing the results with a SMC approach and showing improved chattering rejection capabilities. Moreover, the robustness of the HOSM and CHOSM observers was verified introducing additive noise to the measured variables, obtaining satisfactory results on the estimation of the fuel cell temperature and liquid water fraction. The estimation of the internal water fraction is of special relevance to characterise the fuel cell state-ofhealth as it is an unmeasurable parameter of the system. a)

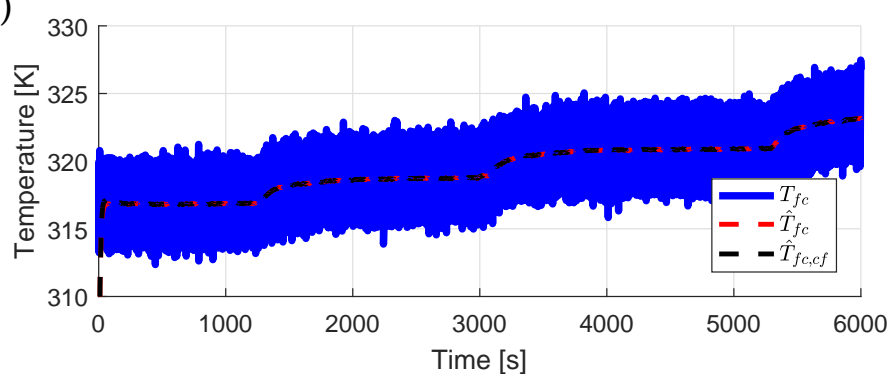

b)

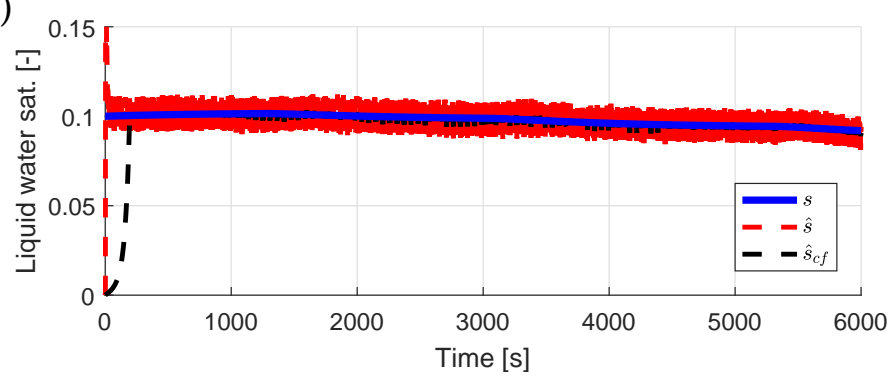

Figure 8: State estimation for the temperature (a) and the liquid water fraction (b) with increased voltage measurement noise

Future work includes deploying the validated observers in stationary and mobile fuel cell applications, such as microgrids and fuel cell powered vehicles, among others. The first stage of the implementation will be to test the behaviour and robustness of the observation strategy under real world conditions. After this, the proper tuning for the observer parameters will be performed for real-time deployment. Then, using the additional system information given by the observer, advanced control strategies will be designed to achieve optimal reference tracking of the internal fuel cell conditions (such as cell temperature and humidity) which are hardly measurable in-situ. By means of using the proposed observation strategy along with advance control solutions, fuel cell applications will be able to operate with a set of adequate internal conditions in order to achieve application-individual lifetime and performance goals. 


\section{Appendix A. FUEL CELL MODEL}

Appendix A.1. Model constants

$$
\begin{aligned}
K_{s} & =\epsilon_{e f f} \cdot d_{C L} \cdot \rho_{l} \cdot K_{\text {sorp }} \\
K_{1} & =\frac{V_{t h} \cdot n_{c e l l}}{m_{f c} \cdot C_{p, f c}} \\
K_{1}^{\prime} & =\frac{1}{m_{f c} \cdot C_{p, f c}} \\
K_{2} & =\frac{\rho_{\text {air }} \cdot A_{\text {inlet }} \cdot C_{p, a i r}}{m_{f c} \cdot C_{p, f c}} \\
K_{3} & =\frac{M_{H_{2} O}}{2 \cdot F \cdot A_{g e o} \cdot K_{s}} \\
K_{4} & =\frac{K_{\text {evap }} \cdot M_{H_{2} O}}{R \cdot A_{\text {pore }} \cdot K_{s}} \\
K_{5} & =\frac{n_{\text {cell }} \cdot R}{\alpha \cdot n \cdot F} \\
K_{6} & =n_{\text {cell }} \cdot R_{\text {ohm }} \\
K_{7} & =n_{\text {cell }}
\end{aligned}
$$

Appendix A.2. Model nonlinear functions

$$
\begin{aligned}
f_{a}\left(T_{f c}, s_{f c}, I_{f c}\right)= & \frac{I_{f c}}{A_{g e o} \cdot i_{0}^{A E C D}} \quad(\mathrm{~A} .10) \\
f_{d}\left(T_{f c}\right)= & \exp \left(\frac{\Delta G^{*}}{R}\right. \\
& \left.\cdot\left(\frac{1}{T^{r e f}}-\frac{1}{T_{f c}}\right)\right) \quad(\mathrm{A} .11) \\
f_{p}\left(T_{f c}\right)= & \left(p_{0}^{\text {sat }} \cdot \exp \left(-\frac{E_{a}}{k_{B} \cdot T_{f c}}\right)-p^{v}\right) \\
& \cdot \frac{K_{\text {evap }}}{101300}(\mathrm{~A} .12) \\
i_{0}^{A E C D}= & 0.21 \cdot i_{0}^{r e f} \cdot a_{c} \cdot \exp \left(f_{d}\right) \\
& \cdot\left(\exp \left(\frac{s_{f c} \cdot 0.01}{s^{o p t}}\right)-1\right) \\
& \cdot \frac{101}{\exp \left(s^{\text {opt }}\right)}(\mathrm{A} .13)
\end{aligned}
$$

\section{Acknowledgements}

This work has been partially funded by the Spanish national project DOVELAR (ref. RTI2018-096001B-C32, MINECO/FEDER), the Spanish State Research Agency through the María de Maeztu Seal of Excellence to IRI (MDM-2016-0656), and AGAUR of Generalitat de Catalunya through the Advanced Control Systems (SAC) group grant (2017 SGR 482).

\section{References}

[1] B. E. Logan, "Peer reviewed: extracting hydrogen and electricity from renewable resources," Environmental Science \& Technology, vol. 38, no. 9, pp. 160A-167A, 2004.

[2] S. H. Jensen, P. H. Larsen, and M. Mogensen, "Hydrogen and synthetic fuel production from renewable energy sources," International Journal of Hydrogen Energy, vol. 32, no. 15, pp. 3253-3257, 2007.

[3] F. Barbir, PEM fuel cells: theory and practice. Elsevier Academic Press, 2013.

[4] N. Yousfi-Steiner, P. Moçotéguy, D. Candusso, D. Hissel, A. Hernandez, and A. Aslanides, "A review on PEM voltage degradation associated with water management: Impacts, influent factors and characterization," Journal of Power Sources, vol. 183, no. 1, pp. 260-274, 2008.

[5] A. Phillips, M. Ulsh, K. Neyerlin, J. Porter, and G. Bender, "Impacts of electrode coating irregularities on polymer electrolyte membrane fuel cell lifetime using quasi in-situ infrared thermography and accelerated stress testing," International Journal of Hydrogen Energy, vol. 43, no. 12, pp. 6390-6399, 2018.

[6] J. Luna, E. Usai, A. Husar, and M. Serra, "Enhancing the efficiency and lifetime of a proton exchange membrane fuel cell using nonlinear model-predictive control with nonlinear observation," IEEE transactions on industrial electronics, vol. 64, no. 8, pp. 6649-6659, 2017.

[7] H. A. Kazem and M. T. Chaichan, "Experimental analysis of the performance characteristics of PEM fuel cells," International Journal of Scientific \& Engineering Research, vol. 7, no. 2, pp. 49-56, 2016.

[8] D. N. Ozen, B. Timurkutluk, and K. Altinisik, "Effects of operation temperature and reactant gas humidity levels on performance of PEM fuel cells," Renewable and Sustainable Energy Reviews, vol. 59, pp. 1298-1306, 2016.

[9] W. He, G. Lin, and T. Van Nguyen, "Diagnostic tool to detect electrode flooding in proton-exchange-membrane fuel cells," AIChE Journal, vol. 49, no. 12, pp. 3221-3228, 2003.

[10] A. Kusoglu, A. Kwong, K. T. Clark, H. P. Gunterman, and A. Z. Weber, "Water uptake of fuel-cell catalyst layers," Journal of The Electrochemical Society, vol. 159, no. 9, pp. F530-F535, 2012.

[11] J. T. Gostick, M. A. Ioannidis, M. W. Fowler, and M. D. Pritzker, "Direct measurement of the capillary pressure characteristics of water-air-gas diffusion layer systems for pem fuel cells," Electrochemistry Communications, vol. 10, no. 10, pp. $1520-1523,2008$.

[12] J. T. Gostick, M. W. Fowler, M. A. Ioannidis, M. D. Pritzker, Y. Volfkovich, and A. Sakars, "Capillary pressure and hydrophilic porosity in gas diffusion layers for polymer electrolyte fuel cells," Journal of Power Sources, vol. 156, no. 2, pp. 375 387, 2006.

[13] K. G. Gallagher, R. M. Darling, T. W. Patterson, and M. L. Perry, "Capillary pressure saturation relations for PEM fuel cell gas diffusion layers," Journal of The Electrochemical Society, vol. 155, no. 11, p. B1225, 2008.

[14] K. Akermi, S. Chouraqui, and B. Boudaa, "Novel smc control design for path following of autonomous vehicles with uncertainties and mismatched disturbances," International Journal of Dynamics and Control, vol. 8, no. 1, pp. 254-268, 2020.

[15] J. Luna, E. Usai, A. Husar, and M. Serra, "Nonlinear observation in fuel cell systems: A comparison between disturbance estimation and high-order sliding-mode techniques," International Journal of Hydrogen Energy, vol. 41, no. 43, pp. $19737-$ $19748,2016$.

[16] M. Piffard, M. Gerard, R. Da Fonseca, P. Massioni, and E. Bideaux, "Sliding mode observer for proton exchange membrane fuel cell: automotive application," Journal of Power Sources, vol. 388, pp. 71-77, 2018.

[17] L. Xiong, P. Li, and J. Wang, "High-order sliding mode control of dfig under unbalanced grid voltage conditions," International Journal of Electrical Power \& Energy Systems, vol. 117, p. $105608,2020$. 
Parameter

Specific heat capacity of the fuel cell stack, $C_{p, f c}$

Fuel cell stack mass, $m_{f c}$

Air density @ $20^{\circ} \mathrm{C}, \rho_{\text {air }}$

Specific heat capacity of air @ $20^{\circ} \mathrm{C}, C_{p, \text { air }}$

Effective cross-section of the cathode housing structure, $A_{\text {inlet }}$

Theoretical potential @ $T^{r e f}=25^{\circ} \mathrm{C}$ and $P^{r e f}=1 \mathrm{~atm}, V_{t h}$

Number of cells in the stack, $n_{\text {cell }}$

Intrinsic exchange current density of $\mathrm{Pt}, i_{0}^{r e f}$

Charge transfer coefficient, $\alpha$

Activation barrier for the ORR on $\mathrm{Pt}, \Delta G^{*}$

Optimal reachable liquid water saturation, $s^{\text {opt }}$

Geometric catalyst surface area, $A_{\text {geo }}$

Partial pressure of oxygen at the cathode, $p_{\mathrm{O}_{2}}$

Effective porosity, $\epsilon_{e f f}$

Liquid water density, $\rho_{l}$

Effective thickness of diffusion media, $d_{e f f}$

Catalyst layer volume, $V_{C L}$

Catalyst layer thickness, $d_{C L}$

Pre-exponential factor, $p_{0}^{\text {sat }}$

Activation energy of evaporation, $E_{a}$

Boltzmann constant, $k_{B}$

Cathode ambient pressure, $P_{a m b}$

Cathode ambient temperature, $T_{a m b}$

Cathode ambient vapour pressure @ $75 \% R H, p^{v}$
Value

Units

$1200 \quad \mathrm{~J} \cdot \mathrm{kg}^{-1} \mathrm{~K}^{-1}$

$0.3 \quad \mathrm{~kg}$

$1.205 \quad \mathrm{~kg} \cdot \mathrm{m}^{-3}$

$1005 \mathrm{~J} \cdot \mathrm{kg}^{-1} \cdot \mathrm{K}^{-1}$

$8.5 e^{-3} \quad \mathrm{~m}^{2}$

$1.13 \mathrm{~V}$

20

$5 e^{-3}$

$\mathrm{A} \cdot \mathrm{m}^{-2}$

0.3

70000

0.1

$22.5 e^{-4}$

$0.21 \cdot P^{r e f}$

0.5

970

$0.41 e^{-3}$

$2.25 e^{-8}$

$0.01 e^{-3}$

$1.196 e^{11}$

0.449

$8.617 e^{-5}$

$1.013 e^{5}$

298

2380
$\mathrm{J} \cdot \mathrm{mol}^{-1}$

$\mathrm{m}^{2}$

$\mathrm{Pa}$

$\mathrm{kg} \cdot \mathrm{m}^{-3}$

$\mathrm{m}$

$\mathrm{m}^{3}$

$\mathrm{m}$

$\mathrm{Pa}$

$\mathrm{eV}$

$\mathrm{eV} \cdot \mathrm{K}^{-1}$

$\mathrm{Pa}$

K

$\mathrm{Pa}$ 
[18] V. Utkin, A. Poznyak, Y. Orlov, and A. Polyakov, "Conventional and high order sliding mode control," Journal of the Franklin Institute, 2020

[19] S. Strahl, A. Husar, P. Puleston, and J. Riera, "Performance improvement by temperature control of an open-cathode PEM fuel cell system," Fuel Cells, vol. 14, no. 3, pp. 466-478, 2014.

[20] U. Pasaogullari and C. Wang, "Liquid water transport in gas diffusion layer of polymer electrolyte fuel cells," Journal of the Electrochemical Society, vol. 151, no. 3, pp. A399-A406, 2004.

[21] S. Strahl and R. Costa-Castelló, "Model-based analysis for the thermal management of open-cathode proton exchange membrane fuel cell systems concerning efficiency and stability," Journal of Process Control, vol. 47, pp. 201-212, 2016.

[22] J. Davila, L. Fridman, A. Pisano, and E. Usai, "Finite-time state observation for non-linear uncertain systems via higherorder sliding modes," International Journal of Control, vol. 82 , no. 8, pp. 1564-1574, 2009.

[23] J. J. E. Slotine and W. Li, Applied nonlinear control, vol. 199, no. 1. Prentice-Hall Englewood Cliffs, NJ, 1991.

[24] A. Levant, "Higher-order sliding modes, differentiation and output-feedback control," International Journal of Control, vol. 76, no. 9-10, pp. 924-941, 2003.

[25] A. Rosales, Y. Shtessel, L. Fridman, and C. Panathula, "Chattering analysis of hosm controlled systems: frequency domain approach," IEEE Transactions on Automatic Control, vol. 62, no. 8, pp. 4109-4115, 2017.

[26] S. Mondal and C. Mahanta, "Chattering free adaptive multivariable sliding mode controller for systems with matched and mismatched uncertainty," ISA transactions, vol. 52, no. 3, pp. 335-341, 2013.

[27] Y. Xiong and M. Saif, "Sliding mode observer for nonlinear uncertain systems," IEEE transactions on Automatic Control, vol. 46, no. 12, pp. 2012-2017, 2001. 